\title{
Examination of Proper Randomness of the Numbers Generated by Rand Corporation (1955) Random Number Table: t-test
}

\author{
Brajendra Kanta Sarmah ${ }^{1}$, Dhritikesh Chakrabarty ${ }^{2}$, \\ Associate Professor, Department of Statistics, Bholanath College, Dhubri, Assam, India ${ }^{1}$ \\ Associate Professor, Department of Statistics, Handique Girls' College, Assam, India ${ }^{2}$
}

\begin{abstract}
Proper randomness of the numbers generated by Rand Corporation has been tested by B.K. Sarmah, D. Chakrabarty and N. Barman, IJESM 5 (1) : January - March, 2015, 97-119 by applying the Chi-square test for testing the significance of difference between observed frequency of each of the digit in the table and the corresponding theoretical (expected) frequency .

In this paper, the randomness of the digits have been tested by applying t-test for amount of deviation of the observed number of occurrences and the theoretical (expected) number of occurrences of the respective digits and hence the numbers.
\end{abstract}

The test shows that the numbers generated by Rand Corporation deviated significantly in most the observations from proper randomness.

KEYWORDS: Random number generated by Rand Corporation, student t-test, testing of randomness.

\section{INTRODUCTION}

Drawing of random sample has been found to be vital or basic necessity in most of the researches and investigations especially of applied sciences. The convenient practical method of selecting a random sample consists of the use of Table of Random Numbers. Existing tables of random numbers, used commonly, are the ones due to Fisher and Yates (Constructed in 1938), L.H.C. Tippett (Constructed in 1927), Kendall and Babington Smith (Constructed in 1939) and Rand Corporation (constructed in 1955)

The random number tables have been subjected to various statistical tests of randomness. These tests have limitations to decide on proper randomness of the numbers occurring in the corresponding tables. As a Consequence it is not guaranteed that the numbers in each of these tables are properly random. This leads to think of testing the proper randomness of the numbers in the tables. In the present study, an attempt has been made to test this. The study, here, has been made on the testing of randomness of the table of numbers constructed by Rand Corporation only. By the existing statistical methods, it is only possible to know whether the randomness of the numbers of a table is proper. It is only possible to know whether the deviation of the degree of its randomness is significant. In order to test the proper randomness of the random numbers table constructed by Rand Corporation, t-test has been applied. The test shows that Rand Corporation's Random numbers table deviate significantly from proper randomness.

\section{MATERIALS AND METHODS}

Rand Corporation random number table consists of a total of one million random digits grouped into $2,00,000$ sets of 5 digited random numbers . Here a sample of 50000 digites are considered out of one million digits for testing Randomness.

To know whether the number in random numbers table of Rand Corporation are proper or not student's t-test for amount of deviation is applied.

Let ' $\mathrm{d}$ ' be the variable denoting the measure of the deviation (amount of deviation) of the observed number of occurrences of the respective digit.

Suppose, $\operatorname{di}(\mathrm{i}=0,1 \ldots .9)$ are independent observed values of the deviation variables. 
ISSN(Online) :2319-8753

ISSN (Print) : 2347-6710

\section{International Journal of Innovative Research in Science, Engineering and Technology}

(An ISO 3297: 2007 Certified Organization)

Vol. 4, Issue 10, October 2015

If the table of number is random then $\mathrm{di}=0$, for all $\mathrm{i}$, in the ideal situation . However, due to chance error, di may assume non zero value.

Thus the values of di's are due to chance error but not due to any assignable error if the table is random.

The chance variables are i.i.d. N.(o, $\sigma)$ variables.

Thus testing of randomness is equivalent to testing of the hypothesis $\mathrm{H}_{0}$

That $\quad E\left(d_{i}\right)=0$, for all $i$,

Let us consider the statistic $\mathrm{t}$ for testing $\mathrm{H}_{\mathrm{o}}$

where,

$$
\text { i.e. } t=\frac{\bar{d}-E(\bar{d})}{\operatorname{S.E} .(\bar{d})} \sim t_{n-1}
$$

We have,

$$
\bar{d}=\frac{1}{n} \sum_{i=1}^{n} d_{i}
$$

$$
\mathrm{E}(\bar{d})=\frac{1}{n} \sum E\left(d_{i}\right)=0
$$

When $\mathrm{H}_{\mathrm{o}}$ is true

Also,

$$
\operatorname{var} \overline{(d)}=\frac{\sigma^{2}}{n}, \sigma^{2} \text { is unknown }
$$

However unbiased estimate of $\sigma^{2}$ is

$$
\begin{aligned}
& s^{2}=\frac{1}{n-1} \sum\left(d_{i}-\bar{d}\right)^{2} \\
= & \frac{1}{n-1}\left[\sum d_{i}{ }^{2}-\frac{\left(\Sigma d_{i}\right)^{2}}{n}\right]
\end{aligned}
$$

Which implies unbiased estimate of

$$
\operatorname{var} \overline{(d)}=\frac{s^{2}}{n}
$$

and $S \cdot D \cdot \overline{(d)}=\frac{s}{\sqrt{n}}$

Therefore statistic $\mathrm{t}$ for testing $\mathrm{H}_{\mathrm{o}}$ becomes

$$
t=\frac{\bar{d}}{s / \sqrt{n}} \text { when } H_{\mathrm{o}} \text { is true and this } \mathrm{t} \text { follows student's t distribution with (n-1) d.f. }
$$

\section{STEPS IN THE METHOD}

In order to test the proper randomness of the numbers of Rand Corporation table one is required to proceed with the following steps:

Step1: In the first step, observe the occurrences of the digits 0 to 9 for first 2000 trails, second 2000 trials

up to $25^{\text {th }} 2000$ trails as shown in the table.

Step2: In the second step, compute the theoretical expected frequencies. This is done by dividing trails i.e. $1^{\text {st }}$ $2000 \ldots \ldots \ldots \ldots$ and $25^{\text {th }} 2000$ trails by 10 assuming that the digit 0 to 9 occurs equal number of times.

Step 3: In the third step, compute the amount of deviation of observed occurrences of digits and expected occurrences of digits.

Step 4: In the fourth step, compute the value of student's $t$ for each of the trails.

Step 5: Compare the value of t-statistic with corresponding theoretical (expected) values.

Step 6: Draw conclusion as per the result obtained in step 5.

\section{RESULTS AND DISCUSSION}

The results obtained on operating the steps (Nos. 1 to 5) on the random numbers table constructed by Rand Corporation have been observed. It is observed from the table that occurrences of digit 0 to 9 are not equal.

\section{CONCLUSION}

From the table prepared for observed frequency of occurrence of digits along with respective expected frequency (shown in bracket) it is observed that the calculated value of $t$ is significant in all most of the cases except 


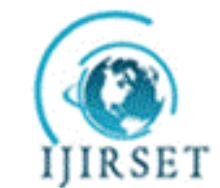 \\ ISSN(Online) :2319-8753 \\ ISSN (Print) : 2347-6710 \\ International Journal of Innovative Research in Science, Engineering and Technology

\author{
(An ISO 3297: 2007 Certified Organization)
}

Vol. 4, Issue 10, October 2015

$3^{\text {rd }}, 7^{\text {th }}, 20^{\text {th }}, 23^{\text {rd }}, 25$ th 2000 trails. That is calculated value of ' $t$ ' have been found to be significant on comparing them with the corresponding theoretical values for most of the cases (trails).

Hence it may be concluded that the table of numbers constructed by Rand Corporation deviates significantly from proper randomness.

Therefore Rand Corporation Random Numbers Table Cannot be treated as properly random, as per results obtained by applying t-test.

TABLE

Observed frequency of occurrence of digits along with the respective expected frequency (Shown in bracket), amount of deviation (di) and the values of students ' $t$ ' statistic from Rand Corporation .

\begin{tabular}{|c|c|c|c|c|c|c|c|c|c|c|c|}
\hline Digits & 0 & 1 & 2 & 3 & 4 & 5 & 6 & 7 & 8 & 9 & $\begin{array}{c}\text { Value } \\
\text { of } t\end{array}$ \\
\hline $1^{\text {st }}$ & 6 & 16 & 16 & 0 & 24 & 11 & 3 & 4 & 16 & 6 & \\
\hline 2000 & $\begin{array}{c}206 \\
(200)\end{array}$ & $\begin{array}{c}184(20 \\
0)\end{array}$ & $\begin{array}{c}184(20 \\
0)\end{array}$ & $\begin{array}{c}200(20 \\
0)\end{array}$ & $224(200$ & 211(200) & $\begin{array}{c}197(20 \\
0)\end{array}$ & 204(200 & $184(200)$ & $\begin{array}{c}206(20 \\
0)\end{array}$ & 4.04 \\
\hline nd & 3 & 19 & 15 & 6 & 15 & 19 & 8 & 5 & 0 & 0 & \\
\hline $\begin{array}{c}2^{\text {nat }} \\
2000\end{array}$ & $\begin{array}{c}197 \\
(200)\end{array}$ & $\begin{array}{c}219 \\
(200)\end{array}$ & $\begin{array}{c}215 \\
(200)\end{array}$ & $\begin{array}{c}206 \\
(200)\end{array}$ & $\begin{array}{c}185 \\
(200)\end{array}$ & $\begin{array}{c}181 \\
(200)\end{array}$ & $\begin{array}{c}192 \\
(200)\end{array}$ & $\begin{array}{c}205 \\
(200)\end{array}$ & $200(200)$ & $\begin{array}{c}200 \\
(200)\end{array}$ & 4.21 \\
\hline $\mathrm{r}^{\mathrm{rd}}$ & 2 & 11 & 3 & 15 & 26 & 2 & 17 & 5 & 2 & 13 & \\
\hline 2000 & $\begin{array}{c}202 \\
(200)\end{array}$ & $\begin{array}{c}189 \\
(200)\end{array}$ & $\begin{array}{c}203 \\
(200)\end{array}$ & $\begin{array}{c}215 \\
(200)\end{array}$ & $\begin{array}{c}226 \\
(200)\end{array}$ & $\begin{array}{c}198 \\
(200)\end{array}$ & $\begin{array}{c}183 \\
(200)\end{array}$ & $\begin{array}{c}195 \\
(200)\end{array}$ & $\begin{array}{c}202 \\
(200)\end{array}$ & $\begin{array}{c}\mathbf{1 8 7} \\
(200)\end{array}$ & $3.21 *$ \\
\hline & 8 & 24 & 18 & 11 & 24 & 4 & 5 & 1 & 8 & 3 & \\
\hline $\begin{array}{c}4 \\
2000\end{array}$ & $\begin{array}{c}192 \\
(200)\end{array}$ & $\begin{array}{c}224 \\
(200)\end{array}$ & $\begin{array}{c}182 \\
(200)\end{array}$ & $\begin{array}{c}189 \\
(200)\end{array}$ & $\begin{array}{c}224 \\
(200)\end{array}$ & $\begin{array}{c}196 \\
(200)\end{array}$ & $\begin{array}{c}205 \\
(200)\end{array}$ & $\begin{array}{c}199 \\
(200)\end{array}$ & $\begin{array}{c}192 \\
(200)\end{array}$ & $\begin{array}{c}197 \\
(200)\end{array}$ & 4.00 \\
\hline & 7 & 7 & 14 & 25 & 13 & 7 & 10 & 14 & 2 & 7 & \\
\hline $\begin{array}{c}5 \\
2000\end{array}$ & $\begin{array}{c}207 \\
(200) \\
\end{array}$ & $\begin{array}{c}207 \\
(200)\end{array}$ & $\begin{array}{c}186 \\
(200)\end{array}$ & $\begin{array}{c}175 \\
(200)\end{array}$ & $\begin{array}{c}213 \\
(200) \\
\end{array}$ & $\begin{array}{c}193 \\
(200) \\
\end{array}$ & $\begin{array}{c}210 \\
(200)\end{array}$ & $\begin{array}{c}214 \\
(200) \\
\end{array}$ & $\begin{array}{c}202 \\
(200) \\
\end{array}$ & $\begin{array}{c}193 \\
(200) \\
\end{array}$ & 5.00 \\
\hline & 8 & 7 & 11 & 15 & 16 & 3 & 12 & 12 & 3 & 3 & \\
\hline $\begin{array}{c}0 \\
2000\end{array}$ & $\begin{array}{c}208 \\
(200) \\
\end{array}$ & $\begin{array}{c}193 \\
(200)\end{array}$ & $\begin{array}{l}\mathbf{1 8 9} \\
(200)\end{array}$ & $\begin{array}{c}215 \\
(200)\end{array}$ & $\begin{array}{c}216 \\
(200)\end{array}$ & $\begin{array}{c}203 \\
(200)\end{array}$ & $\begin{array}{c}188 \\
(200)\end{array}$ & $\begin{array}{c}188 \\
(200)\end{array}$ & $\begin{array}{c}203 \\
(200)\end{array}$ & $\begin{array}{c}197 \\
(200)\end{array}$ & 6.91 \\
\hline & 4 & 4 & 8 & 7 & 1 & 0 & 21 & 11 & 21 & 1 & \\
\hline 2000 & $\begin{array}{c}196 \\
(200) \\
\end{array}$ & $\begin{array}{c}196 \\
(200)\end{array}$ & $\begin{array}{c}192 \\
(200)\end{array}$ & $\begin{array}{c}207 \\
(200)\end{array}$ & $\begin{array}{c}199 \\
(200)\end{array}$ & $\begin{array}{c}200 \\
(200)\end{array}$ & $\begin{array}{c}221 \\
(200)\end{array}$ & $\begin{array}{c}211 \\
(200)\end{array}$ & $\begin{array}{c}179 \\
(200)\end{array}$ & $\begin{array}{c}199 \\
(200)\end{array}$ & $3.28 *$ \\
\hline & 19 & 11 & 19 & 11 & 5 & 17 & 10 & 11 & 7 & 4 & \\
\hline 2000 & $\begin{array}{c}181 \\
(200)\end{array}$ & $\begin{array}{c}211 \\
(200)\end{array}$ & $\begin{array}{c}219 \\
(200)\end{array}$ & $\begin{array}{c}189 \\
(200)\end{array}$ & $\begin{array}{c}195 \\
(200)\end{array}$ & $\begin{array}{c}217 \\
(200)\end{array}$ & $\begin{array}{c}210 \\
(200)\end{array}$ & $\begin{array}{c}189 \\
(200)\end{array}$ & $\begin{array}{c}193 \\
(200)\end{array}$ & $\begin{array}{c}196 \\
(200)\end{array}$ & 7.27 \\
\hline & 9 & 0 & 1 & 10 & 10 & 9 & 19 & 1 & 20 & 1 & \\
\hline $\begin{array}{c}9^{n} \\
2000\end{array}$ & $\begin{array}{c}191 \\
(200)\end{array}$ & $\begin{array}{c}200 \\
(200)\end{array}$ & $\begin{array}{c}199 \\
(200)\end{array}$ & $\begin{array}{c}210 \\
(200)\end{array}$ & $\begin{array}{c}210 \\
(200)\end{array}$ & $\begin{array}{c}191 \\
(200)\end{array}$ & $\begin{array}{c}219 \\
(200)\end{array}$ & $\begin{array}{c}199 \\
(200)\end{array}$ & $\begin{array}{c}180 \\
(200)\end{array}$ & $\begin{array}{c}201 \\
(200)\end{array}$ & 3.59 \\
\hline & 11 & 1 & 19 & 4 & 10 & 16 & 16 & 22 & 15 & 8 & \\
\hline 2000 & $\begin{array}{c}189 \\
(200)\end{array}$ & $\begin{array}{c}199 \\
(200)\end{array}$ & $\begin{array}{c}181 \\
(200)\end{array}$ & $\begin{array}{c}196 \\
(200)\end{array}$ & $\begin{array}{c}190 \\
(200)\end{array}$ & $\begin{array}{c}184 \\
(200)\end{array}$ & $\begin{array}{c}216 \\
(200)\end{array}$ & $\begin{array}{c}222 \\
(200)\end{array}$ & $\begin{array}{c}215 \\
(200)\end{array}$ & $\begin{array}{c}208 \\
(200)\end{array}$ & 5.53 \\
\hline & 31 & 3 & 11 & 8 & 6 & 27 & 13 & 18 & 5 & 22 & \\
\hline 2000 & $\begin{array}{c}169 \\
(200)\end{array}$ & $\begin{array}{c}197 \\
(200)\end{array}$ & $\begin{array}{c}189 \\
(200)\end{array}$ & $\begin{array}{c}192 \\
(200)\end{array}$ & $\begin{array}{c}194 \\
(200)\end{array}$ & $\begin{array}{c}227 \\
(200)\end{array}$ & $\begin{array}{c}187 \\
(200)\end{array}$ & $\begin{array}{c}218 \\
(200)\end{array}$ & $\begin{array}{c}205 \\
(200)\end{array}$ & $\begin{array}{c}222 \\
(200)\end{array}$ & 4.11 \\
\hline $12^{\text {th }}$ & 8 & 0 & 9 & 9 & 9 & 21 & 2 & 28 & 29 & 17 & \\
\hline $\begin{array}{l}12 \\
2000\end{array}$ & $\begin{array}{c}108 \\
(200)\end{array}$ & $\begin{array}{c}200 \\
(200)\end{array}$ & $\begin{array}{c}191 \\
(200)\end{array}$ & $\begin{array}{c}209 \\
(200)\end{array}$ & $\begin{array}{c}191 \\
(200)\end{array}$ & $\begin{array}{c}221 \\
(200)\end{array}$ & $\begin{array}{c}198 \\
(200)\end{array}$ & $\begin{array}{c}228 \\
(200)\end{array}$ & $\begin{array}{c}171 \\
(200)\end{array}$ & $\begin{array}{c}183 \\
(200)\end{array}$ & 3.60 \\
\hline $\begin{array}{c}13^{\text {th }} \\
2000\end{array}$ & $\begin{array}{c}26 \\
174 \\
(200)\end{array}$ & $\begin{array}{c}12 \\
188 \\
(200)\end{array}$ & $\begin{array}{c}23 \\
177 \\
(200)\end{array}$ & $\begin{array}{c}9 \\
209 \\
(200)\end{array}$ & $\begin{array}{c}18 \\
218 \\
(200)\end{array}$ & $\begin{array}{c}15 \\
185 \\
(200)\end{array}$ & $\begin{array}{c}0 \\
200 \\
(200)\end{array}$ & $\begin{array}{c}8 \\
192 \\
(200)\end{array}$ & $\begin{array}{c}34 \\
234 \\
(200)\end{array}$ & $\begin{array}{c}23 \\
223 \\
(200)\end{array}$ & 4.65 \\
\hline
\end{tabular}


IJIRSET

ISSN(Online) :2319-8753

ISSN (Print) : 2347-6710

\section{International Journal of Innovative Research in Science, Engineering and Technology}

(An ISO 3297: 2007 Certified Organization)

Vol. 4, Issue 10, October 2015

\begin{tabular}{|c|c|c|c|c|c|c|c|c|c|c|c|}
\hline $\begin{array}{c}14^{\text {th }} \\
2000\end{array}$ & $\begin{array}{c}11 \\
211 \\
(200)\end{array}$ & $\begin{array}{c}15 \\
185 \\
(200)\end{array}$ & $\begin{array}{c}9 \\
209 \\
(200)\end{array}$ & $\begin{array}{c}3 \\
197 \\
(200)\end{array}$ & $\begin{array}{c}1 \\
201 \\
(200)\end{array}$ & $\begin{array}{c}2 \\
198 \\
(200)\end{array}$ & $\begin{array}{c}12 \\
188 \\
(200)\end{array}$ & $\begin{array}{c}9 \\
209 \\
(200)\end{array}$ & $\begin{array}{c}8 \\
200 \\
(200)\end{array}$ & $\begin{array}{c}6 \\
194 \\
(200)\end{array}$ & 4.85 \\
\hline $\begin{array}{l}15^{\text {th }} \\
2000\end{array}$ & $\begin{array}{c}15 \\
215 \\
(200)\end{array}$ & $\begin{array}{c}16 \\
194 \\
(200)\end{array}$ & $\begin{array}{c}29 \\
171 \\
(200)\end{array}$ & $\begin{array}{c}2 \\
202 \\
(200)\end{array}$ & $\begin{array}{c}7 \\
207 \\
(200)\end{array}$ & $\begin{array}{c}16 \\
216 \\
(200)\end{array}$ & $\begin{array}{c}9 \\
191 \\
(200)\end{array}$ & $\begin{array}{c}7 \\
207 \\
(200)\end{array}$ & $\begin{array}{c}1 \\
199 \\
(200)\end{array}$ & $\begin{array}{c}2 \\
198 \\
(200)\end{array}$ & 3.91 \\
\hline $\begin{array}{c}16^{\text {th }} \\
2000\end{array}$ & $\begin{array}{c}17 \\
217 \\
(200) \\
\end{array}$ & $\begin{array}{c}12 \\
188 \\
(200) \\
\end{array}$ & $\begin{array}{c}6 \\
194 \\
(200) \\
\end{array}$ & $\begin{array}{c}2 \\
202 \\
(200)\end{array}$ & $\begin{array}{c}3 \\
203 \\
(200)\end{array}$ & $\begin{array}{c}13 \\
187 \\
(200) \\
\end{array}$ & $\begin{array}{c}5 \\
195 \\
(200) \\
\end{array}$ & $\begin{array}{c}8 \\
208 \\
(200)\end{array}$ & $\begin{array}{c}6 \\
206 \\
(200)\end{array}$ & $\begin{array}{c}0 \\
200 \\
(200)\end{array}$ & 4.80 \\
\hline $\begin{array}{c}17^{\text {th }} \\
2000\end{array}$ & $\begin{array}{c}14 \\
214 \\
(200)\end{array}$ & $\begin{array}{c}23 \\
177 \\
(200)\end{array}$ & $\begin{array}{c}22 \\
222 \\
(200)\end{array}$ & $\begin{array}{c}17 \\
183 \\
(200)\end{array}$ & $\begin{array}{c}9 \\
209 \\
(200)\end{array}$ & $\begin{array}{c}2 \\
202 \\
(200)\end{array}$ & $\begin{array}{c}7 \\
7 \\
207 \\
(200)\end{array}$ & $\begin{array}{c}2 \\
198 \\
(200)\end{array}$ & $\begin{array}{c}12 \\
188 \\
(200)\end{array}$ & $\begin{array}{c}0 \\
200 \\
(200)\end{array}$ & 4.63 \\
\hline $\begin{array}{c}18^{\text {th }} \\
2000\end{array}$ & $\begin{array}{c}3 \\
203 \\
(200)\end{array}$ & $\begin{array}{c}14 \\
186 \\
(200)\end{array}$ & $\begin{array}{c}8 \\
192 \\
(200)\end{array}$ & $\begin{array}{c}11 \\
211 \\
(200)\end{array}$ & $\begin{array}{c}1 \\
201 \\
(200)\end{array}$ & $\begin{array}{c}15 \\
185 \\
(200)\end{array}$ & $\begin{array}{c}1 \\
201 \\
(200)\end{array}$ & $\begin{array}{c}10 \\
210 \\
(200)\end{array}$ & $\begin{array}{c}9 \\
209 \\
(200)\end{array}$ & $\begin{array}{c}2 \\
202 \\
(200)\end{array}$ & 4.56 \\
\hline $\begin{array}{c}19^{\text {th }} \\
2000\end{array}$ & $\begin{array}{c}25 \\
175 \\
(200)\end{array}$ & $\begin{array}{c}15 \\
215 \\
(200)\end{array}$ & $\begin{array}{c}32 \\
232 \\
(200)\end{array}$ & $\begin{array}{c}30 \\
170 \\
(200)\end{array}$ & $\begin{array}{c}3 \\
197 \\
(200)\end{array}$ & $\begin{array}{c}5 \\
205 \\
(200)\end{array}$ & $\begin{array}{c}1 \\
201 \\
(200)\end{array}$ & $\begin{array}{c}9 \\
191 \\
(200)\end{array}$ & $\begin{array}{c}17 \\
217 \\
(200)\end{array}$ & $\begin{array}{c}3 \\
197 \\
(200)\end{array}$ & 3.91 \\
\hline $\begin{array}{c}20^{\text {th }} \\
2000\end{array}$ & $\begin{array}{c}2 \\
202 \\
(200)\end{array}$ & $\begin{array}{c}3 \\
203 \\
(200) \\
\end{array}$ & $\begin{array}{c}2 \\
198 \\
(200)\end{array}$ & $\begin{array}{c}6 \\
194 \\
(200) \\
\end{array}$ & $\begin{array}{c}1 \\
199 \\
(200) \\
\end{array}$ & $\begin{array}{c}7 \\
207 \\
(200)\end{array}$ & $\begin{array}{c}2 \\
202 \\
(200)\end{array}$ & $\begin{array}{c}3 \\
197 \\
(200)\end{array}$ & $\begin{array}{c}15 \\
185 \\
(200)\end{array}$ & $\begin{array}{c}13 \\
201 \\
(200)\end{array}$ & $3.12^{*}$ \\
\hline $\begin{array}{c}21^{\mathrm{st}} \\
2000\end{array}$ & $\begin{array}{c}25 \\
175 \\
(200)\end{array}$ & $\begin{array}{c}25 \\
225 \\
(200)\end{array}$ & $\begin{array}{c}16 \\
216 \\
(200)\end{array}$ & $\begin{array}{c}8 \\
192 \\
(200)\end{array}$ & $\begin{array}{c}4 \\
196 \\
(200)\end{array}$ & $\begin{array}{c}29 \\
171 \\
(200)\end{array}$ & $\begin{array}{c}9 \\
209 \\
(200)\end{array}$ & $\begin{array}{c}22 \\
222 \\
(200)\end{array}$ & $\begin{array}{c}14 \\
214 \\
(200)\end{array}$ & $\begin{array}{c}20 \\
180 \\
(200)\end{array}$ & 5.75 \\
\hline $\begin{array}{c}22^{\text {nd }} \\
2000\end{array}$ & $\begin{array}{c}7 \\
207 \\
(200)\end{array}$ & $\begin{array}{c}11 \\
211 \\
(200)\end{array}$ & $\begin{array}{c}6 \\
194 \\
(200)\end{array}$ & $\begin{array}{c}7 \\
207 \\
(200)\end{array}$ & $\begin{array}{c}14 \\
214 \\
(200)\end{array}$ & $\begin{array}{c}2 \\
202 \\
(200)\end{array}$ & $\begin{array}{c}5 \\
205 \\
(200)\end{array}$ & $\begin{array}{c}20 \\
180 \\
(200)\end{array}$ & $\begin{array}{c}6 \\
194 \\
(200)\end{array}$ & $\begin{array}{c}14 \\
186 \\
(200)\end{array}$ & 4.32 \\
\hline $\begin{array}{c}23^{\text {rd }} \\
2000\end{array}$ & $\begin{array}{c}23 \\
223 \\
(200)\end{array}$ & $\begin{array}{c}6 \\
206 \\
(200)\end{array}$ & $\begin{array}{c}34 \\
166 \\
(200)\end{array}$ & $\begin{array}{c}2 \\
198 \\
(200)\end{array}$ & $\begin{array}{c}2 \\
202 \\
(200)\end{array}$ & $\begin{array}{c}0 \\
200 \\
(200)\end{array}$ & $\begin{array}{c}3 \\
203 \\
(200)\end{array}$ & $\begin{array}{c}0 \\
200 \\
(200)\end{array}$ & $\begin{array}{c}10 \\
210 \\
(200)\end{array}$ & $\begin{array}{c}8 \\
192 \\
(200)\end{array}$ & $2.25^{*}$ \\
\hline $\begin{array}{c}24^{\text {th }} \\
2000\end{array}$ & $\begin{array}{c}18 \\
182 \\
(200)\end{array}$ & $\begin{array}{c}19 \\
219 \\
(200)\end{array}$ & $\begin{array}{c}14 \\
214 \\
(200)\end{array}$ & $\begin{array}{c}11 \\
211 \\
(200)\end{array}$ & $\begin{array}{c}1 \\
199 \\
(200)\end{array}$ & $\begin{array}{c}7 \\
193 \\
(200)\end{array}$ & $\begin{array}{c}1 \\
199 \\
(200)\end{array}$ & $\begin{array}{c}8 \\
208 \\
(200)\end{array}$ & $\begin{array}{c}29 \\
171 \\
(200)\end{array}$ & $\begin{array}{c}4 \\
204 \\
(200)\end{array}$ & 3.96 \\
\hline $\begin{array}{c}25^{\text {th }} \\
2000\end{array}$ & $\begin{array}{c}4 \\
196 \\
(200)\end{array}$ & $\begin{array}{c}3 \\
203 \\
(200)\end{array}$ & $\begin{array}{c}9 \\
209 \\
(200)\end{array}$ & $\begin{array}{c}29 \\
171 \\
(200)\end{array}$ & $\begin{array}{c}6 \\
194 \\
(200)\end{array}$ & $\begin{array}{c}3 \\
197 \\
(200)\end{array}$ & $\begin{array}{c}14 \\
214 \\
(200)\end{array}$ & $\begin{array}{c}1 \\
201 \\
(200)\end{array}$ & $\begin{array}{c}14 \\
214 \\
(200)\end{array}$ & $\begin{array}{c}1 \\
201 \\
(200)\end{array}$ & $3.14 *$ \\
\hline
\end{tabular}

*Indicates the values which are less than theoretical values of $t_{0.01,8}=3.36$

\section{REFERENCES}

1. Cochran, W.G. (1940): "Sample survey", Cambridge University press

2. Chakrabarty, D. (2004): "A theoretical definition of probability based on common sense", Bulletin of pure and applied sciences, 23 E-2, 343349.

3. Chakrabarty, D. (2005): "Probability;Link between classical definition and Empirical definition", J. Ass. Sc. Soc., 45,13-18

4. Chakrabarty, D. (2006): "Non equally likely outcomes: The classical definition of probability", Bulletin of pure and applied sciences, 25, E-2, 471-481

5. Chakrabarty, D. (2007): "Empirical definition of probability : Special case of its Theoretical definations". Int. J. Agricult. Stats. Sci., 3(1), 261267.

6. Chakrabarty, D. (2008): “Bernolli’s definition of probability: Special case of its Chakrabarty definition”, Int. J. . Agricult. Stats. Sci., 4(1), 23267

7. Cochran (1940): “A survey of Experimental design”, Mimeo, U.S.D.A.

8. Fisher, R. A. (1938): Statistical table for biological, agricultural and medical research, $6^{\text {th }}$ edition (1982), Longman group limilted, England, 37-38 \& 134-139.

9. Kendall, M.G. and B. B. Simith (1938): Randomness and Random sampling numbers, Jour. Roy Sat. Soc., 103, $147-166$. 


\section{International Journal of Innovative Research in Science, Engineering and Technology}

\section{(An ISO 3297: 2007 Certified Organization)}

\section{Vol. 4, Issue 10, October 2015}

10. Rand Corporation (1955): A million random digits free press, Glenoe, III.

11. Snedecor, G.W. and W. G. Cochran (1967): "Statistical methods, Lowa state university press, Ames, Lowa, $6^{\text {th }}$ edition.

12. Tippet (1927). "Random number tables" tracts of computer, No.-15, Cambridge University press.

13. Von Mises R. (1939): "Probability, Statistics and Truth" Macmillian.

14. Sarmah, B.K and Chakrabarty, D (2014): “ Testing of Randomness of Number generated by Fisher and Yates"(Chi-Square test), IJESRT, 3(11), 632-636.

15. Sarmah, B.K and Chakrabarty, D (2014): “ Examination of Proper Randomness of the Number Generated by L.H.C Tippett, (Chi-Square test)JESRT, 3(12), 661-668.

16. Sarmah, B.K and Chakrabarty, D (2015): Examination of Proper Randomness of the Number Generated by Kendall and Babington Smith (Chi-square test). IJESRT 4(2) Febuary/15, 260-282.

17. B.K. Sarmah*, D. Chakrabarty, N. Barman (2015): Examination of Proper Randomness of the Number Generated by Rand Corporation (1955) (Chi-square test), IJESM 5(1): January- March, 2015, 97-119.

18. Sarmah, B.K and Chakrabarty, D (2015): Testing of proper Randomness of the numbers generated by Fisher and Yates (Applying t-test) ABJMI Vol-7 issue -I, Jan- June 2015, P 87-90.

19. Sarmah, B.K. and Chakrabarty D. (2015): Examination of proper Randomness of the numbers generated by L.H.C. Tippett (1927)(t-test) IOSR Journal of Mathematics, Vol-11, Issue-3 May-June,2015 PP.35-37 